\title{
CHARACTERIZATIONS OF WEIGHTED HARDY-RELLICH INEQUALITIES AND THEIR APPLICATIONS
}

\author{
Jun CaO, Yongyang Jin, Shoufeng Shen and Yurong Wu
}

Abstract. Let $\Omega$ be a bounded open domain in $\mathbb{R}^{n}$. We establish characterizations of the weighted Hardy-Rellich inequalities that connect the integrals over $\Omega$ of the first and second derivatives of the considered functions, via some weighted vector-valued Hardy inequalities and weighted dual Hardy inequalities. These characterizations are then applied to derive some new weighted Rellich inequalities with homogenous weights that admit singularities on unit sphere $\mathbb{S}^{n-1}$.

Mathematics subject classification (2010): 26D15, 35A23, 42B37, 35P15.

Keywords and phrases: Hardy inequality, Rellich inequality, best constant, Helmholtz decomposition, homogeneous weight.

\section{REFERENCES}

[1] F. G. AvkHADIEv, Hardy type inequalities in higher dimensions with explicit estimate of constants, Lobachevskii J. Math., 21, (2006), 3-31.

[2] F. G. Avkhadiev, Hardy-Rellich inequalities in domains of the Euclidean space, J. Math. Anal. Appl., 442, (2016), 469-484.

[3] F. G. Avkhadiev and I. K. Shafigullin, Sharp estimates of Hardy constants for domains with special boundary properties, Translation of Izv. Vyssh. Uchebn. Zaved. Mat. 2014, 69-73, Russian Math. (Iz. VUZ), 58, (2014), 58-61.

[4] A. Balinsky, W. D. Evans And R. T. Lewis, The Analysis and Geometry of Hardy's Inequality, Universitext, Springer, Cham, 2015.

[5] G. BARBATIS, Best constants for higher-order Rellich inequalities in $L^{p}(\Omega)$, Math. Z., 255, (2007), 877-896.

[6] G. Barbatis And A. Tertikas, On a class of Rellich inequalities, J. Comput. Appl. Math., 194, (2006), 156-172.

[7] J. Bourgain And H. Brezis, New estimates for the Laplacian, the div-curl, and related Hodge systems, C. R. Math. Acad. Sci. Paris, 338, (2004), 539-543.

[8] P. Caldiroli and R. Musina, Rellich inequalities with weights, Calc. Var., 45, (2012), 147-164.

[9] D. G. CostA, On Hardy-Rellich type inequalities in $\mathbb{R}^{n}$, Appl. Math. Lett., 22, (2009), 902-905.

[10] E. B. DAvieS AND A. M. HinZ, Explicit constants for Rellich inequalities in $L_{p}(\Omega)$, Math. Z., 227, (1998), 511-523.

[11] Y. Di, L. JIANG, S. SHEN AND Y. JIN, A note on a class of Hardy-Rellich type inequalities, J. Inequal. Appl., 84, (2013), 6 pp.

[12] J. Dolbeault, M. Esteban And A. Laptev, Spectral estimates on the sphere, Anal. PDE, 7, (2014), 435-460.

[13] D. E. Edmunds And W. D. Evans, The Rellich inequality, Rev. Mat. Complut., 29, (2016), 511530 .

[14] D. E. Edmunds And R. Hurri-Syrjänen, Weighted Hardy inequalities, J. Math. Anal. Appl., 310, (2005), 424-435.

[15] E. Fabes, O. Mendez And M. Mitrea, Boundary layers on Sobolev-Besov spaces and Poisson's equation for the Laplacian in Lipschitz domains, J. Funct. Anal., 159, (1998), 323-368. 
[16] R. FARWIG AND H. SOHR, Weighted $L^{q}$-theory for the Stokes resolvent in exterior domains, J. Math. Soc. Japan, 49, (1997), 251-288.

[17] A. FröHLICH, The Helmholtz decomposition of weighted $L^{q}$-spaces for Muckenhoupt weights, Navier-Stokes equations and related nonlinear problems, Ann. Univ. Ferrara Sez. VII (N.S.), 46, (2000), 11-19.

[18] G. P. GALDI, An Introduction to the Mathematical Theory of the Navier-Stokes equations. Vol. I: Linearezed Steady Problem, Springer Tracts Nat. Philos. 38, Springer-Verlag, New York, 1994.

[19] P. HajŁaSZ AND P. KosKela, Isoperimetric inequalities and imbedding theorems in irregular domains, J. London Math. Soc. (2), 58, (1998), 425-450.

[20] G. H. HARDY, Note on a theorem of Hilbert, Math. Z., 6, (1920), 314-317.

[21] G. H. Hardy, J. E. Littlewood and G. Pólya, Inequalities, Cambridge Mathematical Library, Cambridge University Press, Cambridge, 1988.

[22] T. Hoffm Ann-Ostenhof AND A. LAPTEV, Hardy inequalities with homogeneous weights, J. Funct. Anal., 268, (2015), 3278-3289.

[23] A. Kufner And L.-E. Persson, Weighted Inequalities of Hardy Type, World Scientific Publishing Co., Inc., River Edge, NJ, 2003.

[24] L. LanZani And E. M. Stein, A note on div curl inequalities, Math. Res. Lett., 12, (2005), 57-61.

[25] J. LEHrB ÄCK, Weighted Hardy inequalities and the size of the boundary, Manuscripta Math., 127, (2008), 249-273.

[26] J. L. LewIS, Uniformly fat sets, Trans. Am. Math. Soc., 308, (1988), 177-196.

[27] E. H. Lieb AND M. Loss, Analysis, Graduate Studies in Mathematics 14, American Mathematical Society, Providence, RI, 2001.

[28] M. Marcus, V. J. Mizel and Y. Pinchover, On the best constant for Hardy's inequality in $R^{n}$, Trans. Amer. Math. Soc., 350 (1998), 3237-3255.

[29] T. Matskewich AND P. E. SobolevskiI, The best possible constant in generalized Hardy's inequality for convex domain in $\mathbb{R}^{n}$, Nonlinear Anal., 28, (1997), 1601-1610.

[30] G. Metafune, M. Sobajima And C. SPina, Weighted Calderón-Zygmund and Rellich inequalities in $L^{p}$, Math. Ann., 361, (2015), 313-366.

[31] B. MucKenhoupt, Weighted norm inequalities for the Hardy maximal function, Trans. Amer. Math. Soc., 165, (1972), 207-226.

[32] J. NEČAS, Sur une méthode pour résoudre les équations aux dérivées partielles du type elliptique, voisine de la variationnelle, Ann. Scuola Norm. Sup. Pisa (3), 16, (1962), 305-326.

[33] N. OKaZAwa, $L^{\infty}$-estimates for Sobolev functions in terms of $\nabla$ and $\Delta$, Math. Z., 262, (2009), $475-515$.

[34] B. Opic And A. Kufner, Hardy-Type Inequalities, Pitman Research Notes in Mathematics Series 219, Longman Scientific Technical, Harlow, 1990.

[35] M. P. Owen, The Hardy-Rellich inequality for polyharmonic operators, Proc. Roy. Soc. Edinburgh Sect. A, 129 (1999), 825-839.

[36] F. RELLICH, Halbbeschränkte Differentialoperatoren Höherer Ordnung, Proceedings of the International Congress of Mathematicians, 1954, Amsterdam, vol. III, pp. 243-250, Erven P. Noordhoff N.V., Groningen; North-Holland Publishing Co., Amsterdam, 1956.

[37] A. Tertikas And N. B. Zographopoulos, Best constants in the Hardy-Rellich inequalities and related improvements, Adv. Math., 209, (2007), 407-459. 